\title{
Application of Collaborative Nursing Model in Elderly Diabetes Care
}

\author{
Yuanyuan Zhang, Xin Wang* \\ Department of Endocrinology, Shaanxi Provincial People’s Hospital, 256 Youyi West Road, Xi’an, China.
}

How to cite this paper: Yuanyuan Zhang, Xin Wang. (2021) Application of Collaborative Nursing Model in Elderly Diabetes Care. International Journal of Clinical and Experimental Medicine Research, 5(3), 330-334.

DOI: 10.26855/ijcemr.2021.07.015

Received: May 28, 2021

Accepted: June 21, 2021

Published: June 24, 2021

*Corresponding author: Xin Wang, Department of Endocrinology, Shaanxi Provincial People's Hospital, 256 Youyi West Road, Xi'an, China.

\begin{abstract}
Objective: To explore the application of collaborative nursing model in elderly diabetes care. Methods: 100 cases of elderly patients with diabetes in our hospital from February 2020 to February 2021 were selected retrospectively. According to the nursing methods, they were divided into collaborative nursing model group and conventional nursing model group, with 50 cases in each group. The blood glucose level, treatment compliance, psychological state and quality of life of the two groups before and after nursing were statistically analyzed. Results: After nursing, the FPG, 2hPG and HbA1c in the collaborative nursing model group were lower than those in the conventional nursing model group $(P<0.05)$. After nursing, the scores of drug, blood glucose, diet, exercise and the total score of treatment compliance in the collaborative nursing model group were higher than those in the conventional nursing model group $(P<0.05)$. After nursing, the SAS score and SDS score of the collaborative nursing model group were lower than those of the conventional nursing model group $(P<0.05)$, and the SF-36 score was higher than that of the conventional nursing model group $(P<0.05)$. Conclusion: The application effect of collaborative nursing mode in elderly diabetes care is better than that of conventional nursing mode.
\end{abstract}

\section{Keywords}

Routine Nursing Mode, Collaborative Nursing Model, Old Age, Diabetes, Blood Glucose, Treatment Compliance, Mentality, Quality of Life

Diabetes is a disease related to many factors, such as heredity, endocrine, immunity and spirit. It is more common in the middle-aged and elderly people. The disease has a long course and many influencing factors. The development and outcome of the disease are not easy to control. Patients often cannot complete the drug treatment correctly, and lack of scientific and reasonable diet and exercise, which seriously affects the treatment effect of diabetes. Relevant medical research results show that the older the diabetic patients are, the longer the course of disease is, the worse the treatment compliance is, which aggravates the difficulty of treatment for elderly diabetic patients [1]. Therefore, it is of great significance to explore a more practical nursing model for elderly diabetes. This study explored the application of collaborative nursing model in elderly diabetes care.

\section{Materials and methods}

\subsection{General information}

100 cases of elderly patients with diabetes in our hospital from February 2020 to February 2021 were selected retrospectively. According to the nursing methods, they were divided into collaborative nursing model group and 
conventional nursing model group. In the collaborative nursing model group, 50 patients were aged from 31 to 83 years old, with an average of (56.24 2.5) years \pm There were 20 females (40.00\%) and 30 males (60.00\%). In terms of course of disease, 28 cases (56.00\%) were 3-15 years old and 22 cases (44.00\%) were 16-30 years old; in terms of accompanying symptoms, 11 cases (22.00\%) had peripheral neuropathy, 10 cases $(20.00 \%)$ had retinopathy, 8 cases $(16.00 \%)$ had diabetic foot, and 5 cases $(10.00 \%)$ had diabetic nephropathy; In terms of educational level, 12 cases $(24.00 \%)$ were primary school or below, 17 cases (34.00\%) were junior high school, 8 cases (16.00\%) were senior high school or technical secondary school, and 13 cases (26.00\%) were junior college or above. In the routine nursing group, 50 patients were aged from 32 to 84 years old, with an average age of (57.63 $1 \pm$ ). There were 21 females (42.00\%) and 29 males (58.00\%). In terms of course of disease, 27 cases (54.00\%) were 3-15 years old and 23 cases (46.00\%) were 16-30 years old; In terms of accompanying symptoms, 12 cases (24.00\%) had peripheral neuropathy, 11 cases (22.00\%) had retinopathy, 7 cases (14.00\%) had diabetic foot, and 4 cases (8.00\%) had diabetic nephropathy; in terms of educational level, 11 cases $(22.00 \%)$ were primary school or below, 16 cases (32.00\%) were junior high school, 9 cases (18.00\%) were senior high school or technical secondary school, and 14 cases $(28.00 \%)$ were junior college or above. There was no significant difference in general information between the two groups $(\mathrm{P}>0.05)$. This study was approved by the medical ethics committee of our hospital, and all patients had informed consent.

\subsection{Inclusion and exclusion criteria}

Inclusive criteria: 1) all patients met the diagnostic criteria of diabetes [2]; 2) Age $\geq 60$ years old; 3) All the patients had normal consciousness and signed the consent form with knowledge. Exclusion criteria: 1) Patients complicated with severe organic diseases; 2) Patients with history of mental disorder and language expression disorder; 3) Patients whose curative effect cannot be effectively evaluated; 4) Patients without complete clinical data; 5) The liver and kidney function and cardiopulmonary function are seriously deficient.

\subsection{Methods}

\subsubsection{Routine nursing model group}

1) Diet nursing: nurses should do a good job in health education for patients, eat less and more meals, eat regularly and quantitatively, and follow the diet principle of low sugar, low fat, appropriate protein, high cellulose and high vitamin; 2) Proper exercise: proper exercise can enhance the body's immunity, increase the body's sensitivity to insulin and control blood sugar. Guide patients to actively participate in aerobic exercise, such as jogging, yoga, etc.

\subsubsection{Collaborative nursing model group}

1) Assess and determine the educational needs of patients and their families. Actively communicate with patients and their families, understand and track the nursing needs of patients at any time. 2) We should establish educational goals together. According to the evaluation data of patients and their families, coordinate with patients and their families and jointly formulate education objectives. The goals are divided into short-term and long-term goals. 3) Choose educational methods. According to the patient's age, occupation, cultural quality and other factors, choose appropriate education methods, and through the establishment of mutual trust between doctors and patients, make the patient from dependence to joint participation. There are mainly three kinds of education methods: auditory education, visual education and tactile education. Hearing education mainly aimed at patients with mild illness, good understanding and good hearing. Nurses explained blood glucose monitoring, insulin injection technology and Exercise Diet face to face; Visual education mainly aims at patients with good eyesight who are not interested in teaching health education methods to boring nurses. Pictures, slides, food models, specimens and other teaching aids are used to teach diabetes knowledge; Tactile education is mainly aimed at patients with mild illness and good physical strength. Nurses teach them massage techniques of feet, legs and other parts of the body, and encourage patients to massage and check each other. 4) Carry out education plan cooperatively, including mutual education among patients and cooperation education among family members. The questions of diabetic patients are supervised and encouraged by each other through mutual supervision of blood glucose monitoring and exchange of personal experience and experience; Family members of patients actively cooperate with doctors in treatment, assist nurses in guiding patients' behaviors, and act as a communication bridge between nurses and patients to maximize patient compliance. 


\subsection{Observation index}

The patients were followed up for 1 month. 1) Blood glucose level. Fasting blood glucose (FPG) and 2-hour postprandial blood glucose (2hPG) were measured by glucose oxidase method before and after nursing, and glycosylated hemoglobin (HbA1c) was measured by ion exchange liquid chromatography; 2) Treatment compliance: before and after the intervention, the patients in the two groups filled in the questionnaire of treatment compliance designed by Bai Xing. The questionnaire referred to the Morisky patient compliance evaluation method introduced by Li Lina and others, including diet, exercise, drug treatment and blood glucose monitoring. The questionnaire includes 20 questions, each of which has 4 options. Each question has a score of $0-4$, a total of 80 . The higher the score is, the better the patient's compliance is [3]; 3) Mental state. Before and after the intervention, self rating Depression Scale (SDS) and self rating Anxiety Scale (SAS) were used to evaluate the emotion of the two groups. SDS consists of 20 items. Each item is scored according to grade 1-4. The total score is converted into depression index. The cumulative score of each item in depression index is 80 (the highest score). No depression was defined as index $<0.50$, and mild to mild depression was defined as index 0.50-0.59; 0.60-0.69 was moderate depression; 70 was severe depression. SAS scale contains 20 items, each item is scored according to grade 1-4, the evaluation result (initial score) is multiplied by 1.25, then the whole number is taken to get the standard score, and the standard score $<50$ is no anxiety; The scores of 50-59 were mild to mild anxiety, 60-69 were moderate anxiety, and $>70$ were severe anxiety [4]; 4) Quality of life. SF-36 is a universal health scale, which includes 8 dimensions: physiological function, physiological function, physical pain, general health, energy, social function, emotional function and mental health. The scale also includes health change indicators, which are used to evaluate the overall change of health status in the past year. The full score of eight dimensions is 100 . The higher the score is, the higher the quality of life is [5].

\subsection{Statistical analysis}

Spss20.0 statistical software was used for analysis, the measurement data was expressed with ( ), the comparison was performed with $t$ test, the repeated measurement data was analyzed with ANOVA, the count data was expressed with rate (\%), the comparison was performed with chi square test, $\mathrm{P}<0.05$ was considered as statistically significant.

\section{Results}

\subsection{Comparison of blood glucose level between two groups before and after nursing}

The FPG, 2hPG and HbA1c of two groups after nursing were lower than those before nursing $(P<0.05)$; After nursing, the FPG, 2hPG and HbA1c in the collaborative nursing model group were lower than those in the conventional nursing model group $(P<0.05)$ (see Table 1$)$.

Table 1. Comparison of blood glucose level between two groups before and after nursing $(\bar{x} \pm s)$

\begin{tabular}{cccccc}
\hline groups & $\mathrm{n}$ & time & FPG $(\mathrm{mmol} / \mathrm{L})$ & 2hPG $(\mathrm{mmol} / \mathrm{L})$ & $\mathrm{HbA} 1 \mathrm{c}(\%)$ \\
\hline \multirow{2}{*}{ collaborative nursing model group } & \multirow{2}{*}{50} & before nursing & $9.25 \pm 1.11$ & $13.51 \pm 1.78$ & $7.83 \pm 0.90$ \\
& & after nursing & $6.10 \pm 1.30$ & $8.20 \pm 1.31$ & $5.70 \pm 1.11$ \\
\multirow{2}{*}{ conventional nursing model group } & \multirow{2}{*}{50} & before nursing & $9.24 \pm 1.30$ & $12.75 \pm 2.70$ & $8.20 \pm 1.00$ \\
& & after nursing & $7.18 \pm 0.90$ & $10.20 \pm 1.67$ & $6.55 \pm 1.08$ \\
\hline
\end{tabular}

\subsection{Comparison of treatment compliance between two groups before and after nursing}

The scores of drug, blood glucose, diet, exercise and the total score of treatment compliance of the two groups after nursing were higher than those before nursing $(P<0.05)$; After nursing, the scores of drug, blood glucose, diet, exercise and the total score of treatment compliance in the collaborative nursing model group were higher than those in the conventional nursing model group $(P<0.05)$ (see Table 2$)$.

Table 2. Comparison of treatment compliance between two groups before and after nursing (scores, $\bar{x} \pm s$ )

\begin{tabular}{cccccc}
\hline groups & $\mathrm{n}$ & time & drug & blood glucose & diet \\
\hline \multirow{2}{*}{ collaborative nursing model group } & \multirow{2}{*}{50} & before nursing & $8.95 \pm 1.26$ & $8.30 \pm 1.57$ & $9.73 \pm 1.92$ \\
& & after nursing & $17.17 \pm 1.91$ & $17.55 \pm 1.80$ & $14.91 \pm 1.83$ \\
\multirow{2}{*}{ conventional nursing model group } & \multirow{2}{*}{50} & before nursing & $9.32 \pm 1.30$ & $9.91 \pm 1.60$ & $9.55 \pm 1.92$ \\
& & after nursing & $12.76 \pm 1.54$ & $12.86 \pm 1.58$ & $12.02 \pm 1.58$ \\
\hline
\end{tabular}


Table 2 (Continued). Comparison of treatment compliance between the two groups before and after nursing (scores, $\bar{x} \pm s$ )

\begin{tabular}{ccccc}
\hline groups & $\mathrm{n}$ & time & exercise & total scores \\
\hline \multirow{2}{*}{ collaborative nursing model group } & \multirow{2}{*}{50} & before nursing & $8.92 \pm 1.62$ & $35.92 \pm 5.17$ \\
& & after nursing & $17.53 \pm 2.07$ & $67.20 \pm 3.51$ \\
conventional nursing model group & \multirow{2}{*}{50} & before nursing & $9.43 \pm 1.40$ & $38.24 \pm 5.28$ \\
& & after nursing & $12.14 \pm 1.80$ & $49.81 \pm 3.22$ \\
\hline
\end{tabular}

\subsection{Comparison of psychological status and quality of life between the two groups before and after nursing}

The SAS score and SDS score of two groups after nursing were lower than before nursing $(\mathrm{P}<0.05)$, the SF-36 score were higher than before nursing $(P<0.05)$; After nursing, SAS score and SDS score of the collaborative nursing model group were lower than those of the conventional nursing model group $(P<0.05)$, and the SF-36 score was higher than that of the conventional nursing model group $(P<0.05)$ (see Table 3$)$.

Table 3. Comparison of psychological status and quality of life between the two groups before and after nursing (scores, $\bar{x} \pm s$ )

\begin{tabular}{cccccc}
\hline groups & $\mathrm{n}$ & time & SAS scores & SDS scores & SF-36 scores \\
\hline \multirow{2}{*}{ collaborative nursing model group } & \multirow{2}{*}{50} & before nursing & $54.50 \pm 9.77$ & $0.60 \pm 0.11$ & $51.97 \pm 9.96$ \\
& & after nursing & $40.52 \pm 7.82$ & $0.43 \pm 0.10$ & $80.00 \pm 14.86$ \\
\multirow{2}{*}{ conventional nursing model group } & \multirow{2}{*}{50} & before nursing & $55.88 \pm 9.64$ & $0.61 \pm 0.14$ & $52.77 \pm 9.16$ \\
& & after nursing & $47.55 \pm 9.20$ & $0.58 \pm 0.11$ & $73.97 \pm 13.77$ \\
\hline
\end{tabular}

\section{Discussion}

With the development of medicine, modern nursing is gradually changing from patient centered responsibility nursing mode to human centered holistic nursing mode. The collaborative nursing mode is a new nursing mode at home and abroad, which breaks the single traditional nursing mode. In terms of personnel allocation, nurses, patients and patients' families are closely linked together to form a "multi-person" model of a small group. Based on the responsibility system, the patients and their families are mobilized to participate actively. In terms of nursing content, the psychological nursing, out of hospital guidance and supervision and various health education of patients are combined. This nursing mode fully mobilized the enthusiasm of the patients, the degree of family cooperation was higher, the patients were more compliant in the treatment process, the mental bad mood decreased, and the comprehensive satisfaction was higher [6].

Relevant medical research shows that the application of collaborative nursing mode for elderly patients with diabetes can not only effectively improve the negative psychology of patients, but also facilitate the control of blood glucose and improve the quality of life of patients, which has application and promotion value in clinical practice [7]. The results showed that after nursing, the FPG, 2hPG and HbA1c in the collaborative nursing model group were lower than those in the conventional nursing model group $(P<0.05)$, which was consistent with the above research results, indicating that collaborative nursing played a positive role in blood glucose control of patients with type 2 diabetes. The reason is that with the change of modern medical model and the improvement of people's health awareness, the purpose of treatment is not only to improve the survival rate and prolong the survival time of patients, but also to improve their quality of life. Collaborative nursing model is a new type of nursing model, which emphasizes nurses as educators and supporters of medical care, and fully reflects the importance of patients' participation in nursing work. Collaborative nursing includes not only responsibility nursing, but also psychological nursing and health education. It encourages patients to participate in nursing work and clinical treatment, gives full play to patients' self-care ability, and improves their enthusiasm and initiative in the process of treatment.

Relevant medical research shows that collaborative nursing mode can improve the treatment compliance of elderly patients with diabetes [8]. The results showed that after nursing, the scores of drug, blood glucose, diet, exercise and treatment compliance in the collaborative nursing model group were higher than those in the conventional nursing model group $(P<0.05)$, which was consistent with the above research results. The reason is the collaborative nursing model, which adopts personalized nursing according to the patient's age, occupation, education level and physical health level, and changes the past simple lecture and preaching model into the joint participation model, which fully mobilizes the patient's enthusiasm for participation. The family members of the patients are the closest and the longest companions of the patients with diabetes. We have strengthened the education of the family 
members, so that they can actively participate in the collaborative nursing, supervise the patients more conveniently and timely, monitor the blood sugar, moderate exercise, regular diet and so on. The joint participation of patients, their families and nurses reflects the holistic nature of scientific nursing. Collaborative nursing model is easier to mobilize the enthusiasm of patients and their families to participate in the treatment, improve patients' treatment mentality and enhance patients' treatment compliance.

The collaborative nursing model has a great effect on improving the emotion of elderly patients with diabetes. Elderly patients with diabetes are prone to depression and anxiety due to poor blood glucose control, multiple complications, physical diseases and heavy economic burden. Bad mood will make patients lose confidence in the treatment and nursing of the disease, and then affect the prognosis of diabetes [9]. The results showed that after nursing, the SAS score and SDS score of the collaborative nursing model group were lower than those of the conventional nursing model group $(P<0.05)$, and the SF-36 score was higher than that of the conventional nursing model group $(P<0.05)$. It shows that the collaborative nursing mode can make the elderly patients have a better state of mind and mood, and achieve a virtuous circle of improvement and mental pleasure in a relatively relaxed treatment atmosphere. The reason is to adopt the human-centered collaborative nursing mode for nursing intervention. Through holding various colorful health education and psychological counseling activities, patients can have a clearer understanding of their own diseases and understand the sufficiency and necessity of healthy mentality for disease control. At the same time, the mutual supervision and encouragement between patients, and the support and company of family members reduced the patients' depression and anxiety [10].

In conclusion, the application effect of collaborative nursing mode in elderly diabetes care is better than that of conventional nursing mode, which is worthy of promotion.

\section{References}

[1] Guo Weiwei, Liu Junmiao. (2021). Improvement of blood glucose index and treatment compliance by implementing collaborative nursing mode in elderly patients with diabetes [J]. Diabetes Tiandi, 2021, 18(2): 177-178.

[2] Fan yuan. (2021). Application effect of collaborative nursing model in elderly diabetes care [J]. Diabetes World, 2021, 18(4): 14.

[3] Tong Jie. (2020). Observation on the application of Patient-Centered Collaborative Nursing Model in elderly patients with diabetes [J]. Forum on Primary Medicine, 2020, 24(5): 728-729.

[4] Yang Lina, Han Baoru, Wu Ying. (2020). Effect of collaborative nursing model on medication compliance of elderly patients with diabetes [J]. Diabetes New World, 2020, 23(7): 88-89.

[5] Wang Guangling. (2020). Analysis of clinical application of collaborative nursing model in elderly diabetes care [J]. Diabetes world, 2020, 17(9): 266.

[6] Deng Yuanyuan. (2020). Application effect of collaborative nursing model in elderly patients with diabetes [J]. Chinese medical guide, 2020, 18(34): 196-197.

[7] Hou Junling. (2020). Observation on clinical effect of collaborative nursing model in elderly patients with diabetes [J]. Heilongjiang Medical Science, 2020, 43(6): 153-154.

[8] Qu Hui. (2020). Value analysis of collaborative nursing model in elderly diabetes care [J]. Diabetes World, 2020, $17(5): 47$.

[9] Peng Huaiying. (2020). Study on application effect of collaborative nursing mode in elderly diabetes care [J]. Medical Aesthetics and Cosmetology, 2020, 29(4): 128.

[10] Zhang Jun. (2019). Discussion on the implementation effect of collaborative nursing mode in elderly diabetes care [J]. Chinese Medical Guide, 2019, 17(33): 262-263. 